\title{
L'《argument économique » dans l'aide à la décision en politique environnementale et son évanescence : réflexions à partir du cas des «boues rouges » de Gardanne
}

\author{
Yves Meinard $^{1, *}$ et Juliette Rouchier ${ }^{2}$ \\ 1 Aide à la décision, CNRS, UMR7243 LAMSADE, Université Paris-Dauphine, PSL Research University, Paris, France \\ 2 Économie, CNRS, UMR7243 LAMSADE, Université Paris-Dauphine, PSL Research University, Paris, France
}

Reçu le 26 janvier 2018. Accepté le 26 février 2019

Le vocabulaire et la normativité économiques sont désormais omniprésents dans le domaine des politiques d'environnement. Mais, au-delà des apparences, quels rôles jouent vraiment dans la prise de décision publique ces références aux modes de raisonnement de l'économiste? C'est la question sur laquelle se sont penchés deux économistes en étudiant la controverse relative aux «boues rouges » de Gardanne. Ils nous montrent que l'argument économique, qui prend la forme de la préservation des emplois, y apparaît d'autorité. Au-delà de leur appel à davantage de rigueur dans la définition de tels arguments, ces auteurs font une intéressante contribution à un champ de recherche aujourd'hui dynamique - dont NSS s'est fait l'écho de longue date, comme l'atteste, par exemple, l'article de Y. Laurans et al. (NSS, 9, 2).

La Rédaction

\begin{abstract}
Résumé - Nous analysons des arguments économiques utilisés dans des prises de décision en politique publique environnementale. Pour cela, nous appliquons une méthodologie d'étude de cas à la question des boues rouges de Gardanne. Notre étude met en évidence des « aides » à la décision qui prétendent s'appuyer sur la science économique, mais échouent à remplir des critères minimaux de rigueur et d'objectivité. Nous utilisons cette étude de cas pour formuler des idées que des études ultérieures pourront tester: (i) l'aide à la décision en politique environnementale ne s'appuie que très peu sur la science économique ; (ii) pourtant elle est submergée par le vocabulaire économique, ce qui suggère (iii) qu'en se donnant des atours scientifiques illusoires, les prétendues (selon les critères proposés ici) aides à la décision usant de ce vocabulaire économique risquent de mettre à mal la légitimité des politiques environnementales au lieu de participer à les justifier.
\end{abstract}

Mots-clés : environnement / gouvernance / aide à la décision / science économique / arguments

\begin{abstract}
The elusiveness of 'economic arguments' in environmental policy decision aiding. In this paper, we investigate various so-called 'decision aiding' practices based on 'economic arguments' used to design and implement environmental public policies. We analyse these practices to determine whether they truly provide an aid to decision-making. We tackle this issue using a case-based inquiry applied to the 'red muds' of Gardanne. This case exemplifies practices, which claim to take advantage of economic science, but are in fact based on fallacious rationales incapable of meeting even minimal rigorousness and scientificity requirements. We do not claim to produce quantitative evidence that our observations are generalizable. Rather, we claim that our empirical elements can be used to articulate ideas whose generality should be investigated in future quantitative studies: (i) decision aiding, when applied to environmental public policies, is barely, if at all, based on economic science; (ii) it is nevertheless overrun with economic vocabulary, which suggests that (iii) the legitimacy of environmental public policies can be undermined by decision aiding practices which, for lack of a robust anchorage in economic science, weaken the possible justification of these policies.
\end{abstract}

Keywords: environment / governance / decision-aid / economics / arguments

\footnotetext{
* Auteur correspondant : yves.meinard@lamsade.dauphine.fr
} 
Ce texte s'intéresse à l' «aide à la décision» en politique publique environnementale ${ }^{1}$. Nous prenons la notion d'aide à la décision en un sens très large, désignant l'appui qui est pris, à différentes étapes de l'élaboration et de la mise en œuvre de ces politiques, par différents acteurs et sous différentes formes, sur des arguments stricto sensu (en un sens de cette expression qu'il nous faudra préciser) afin d'apporter des améliorations à ces politiques. La notion d'aide à la décision ainsi définie a ipso facto une dimension normative. Cette dimension normative jouera un rôle très important dans notre raisonnement, en nous permettant d'opposer des aides à la décision à proprement parler et des prétendues aides à la décision. Naturellement, cet usage normatif, qui nous imposera de clarifier le cadre normatif que nous présupposons, détermine les limites de la validité de notre raisonnement.

Dans ce cadre, nous identifierons et analyserons les « arguments économiques » entourant l'élaboration et la mise en œuvre d'une politique environnementale en vue d'établir si ce recours à des « arguments économiques » peut être qualifié d'aide à la décision à proprement parler (c'està-dire s'appuyant sur des éléments de connaissance).

Notre méthodologie pour cela relèvera de l'étude de cas et nous l'appliquerons à la question des «boues rouges » de Gardanne sur la base de données de terrain (observations et enquêtes). Nous nous appuierons sur cette étude pour mettre en évidence des problèmes qui, nous en faisons l'hypothèse, présentent une certaine généralité. Cependant, la démonstration de cette généralité dépasse les ambitions du présent article dont la méthodologie ne relève ni de la méta-analyse ni de l'étude statistique.

Nous souhaitons montrer que, quand des « arguments économiques » sont utilisés dans le cadre de politiques environnementales, il est souvent douteux qu'il s'agisse vraiment d'arguments - du moins si l'on comprend le terme «argument» en lui associant des exigences normatives. Afin d'étayer ce jugement sur des bases claires et rigoureuses, nous proposons trois critères pour distinguer les éléments de discours qui méritent d'être appelés «arguments», au sens normatif qui nous intéresse: transparence, cadrage, pertinence. Parce que ces critères n'apparaissent pas respectés dans le cas que nous étudions, les «arguments économiques » impliqués ne sont pas des arguments stricto sensu, et les activités qui les mobilisent ne fournissent pas, par conséquent, une aide à la décision stricto sensu.

\footnotetext{
${ }^{1}$ Ce travail s'appuie sur une enquête menée en 2016, reposant sur des entretiens, des observations, la participation à des comités scientifiques et la lecture de nombreux rapports d'expertise ainsi que sur un travail collaboratif d'élaboration conceptuelle de longue haleine des deux auteurs.
}

La suite de ce texte est divisée en trois parties. Nous présentons tout d'abord le cadre conceptuel qui est le nôtre. La partie suivante est consacrée à l'exploration de notre cas d'étude: les boues rouges. Nous concluons enfin brièvement.

\section{Cadre conceptuel}

\section{Policy analytics: prérequis normatifs}

La présente étude assume un positionnement normatif, et plus précisément prescriptif: nous cherchons à produire des recommandations. Il est donc nécessaire de clarifier le cadre normatif qui nous guide. Nous nous référons pour cela à la notion de "policy analytics», introduite par Tsoukiàs et al. (2013). Il s'agit d'un cadre normatif qui stipule que, pour mériter d'être appelée «aide à la décision» au sens strict, toute activité qui prétend à ce titre en matière de politique publique doit : (i) apporter une plus-value démontrable aux décisions ; (ii) être fondée sur la science; (iii) être opérationnelle; (iv) être légitimatrice. Il s'agit clairement et explicitement d'un cadre normatif, puisque la policy analytics explicite des critères qui ont vocation à permettre d'évaluer ex post un processus d'aide à la décision donné ou d'aiguiller ex ante le déploiement d'un tel processus.

Dans le présent travail, nous ne cherchons pas à démontrer le bien-fondé de ce cadre normatif. Nous admettons ce bien-fondé et assumons le fait que notre raisonnement et nos conclusions sont d'une validité strictement limitée par l'acceptation de ce bien-fondé.

Nous nous concentrerons dans ce texte sur la quatrième exigence de ce cadre : l'exigence de légitimation. Cette focalisation découle du choix de notre objet: l'«argument économique». En effet, il existe un lien conceptuel fort entre la notion de légitimité et celle d'argument. L'exposé complet de cette articulation n'est pas l'objet de ce texte. Contentons-nous d'en rappeler les grandes lignes.

\section{Légitimation et acceptabilité}

La notion de légitimité est d'une grande complexité et on en trouve des explicitations très diverses, inscrites dans des cadres conceptuels eux-mêmes très différents. La figure 1 en présente un éventail partiel mais éclairant pour notre propos. Il est classique en science politique de distinguer: d'un côté, les théories qui définissent la légitimité d'une politique uniquement sur la base de la procédure qui a présidé à sa mise en place (B. Légitimité par les procédures) et, de l'autre, les théories qui définissent la légitimité uniquement sur la base des résultats auxquels ces procédures donnent lieu, autrement dit sur la base des caractéristiques des politiques 
Qu'est-ce que l'évaluateur doit examiner pour établir si une politique est légitime?

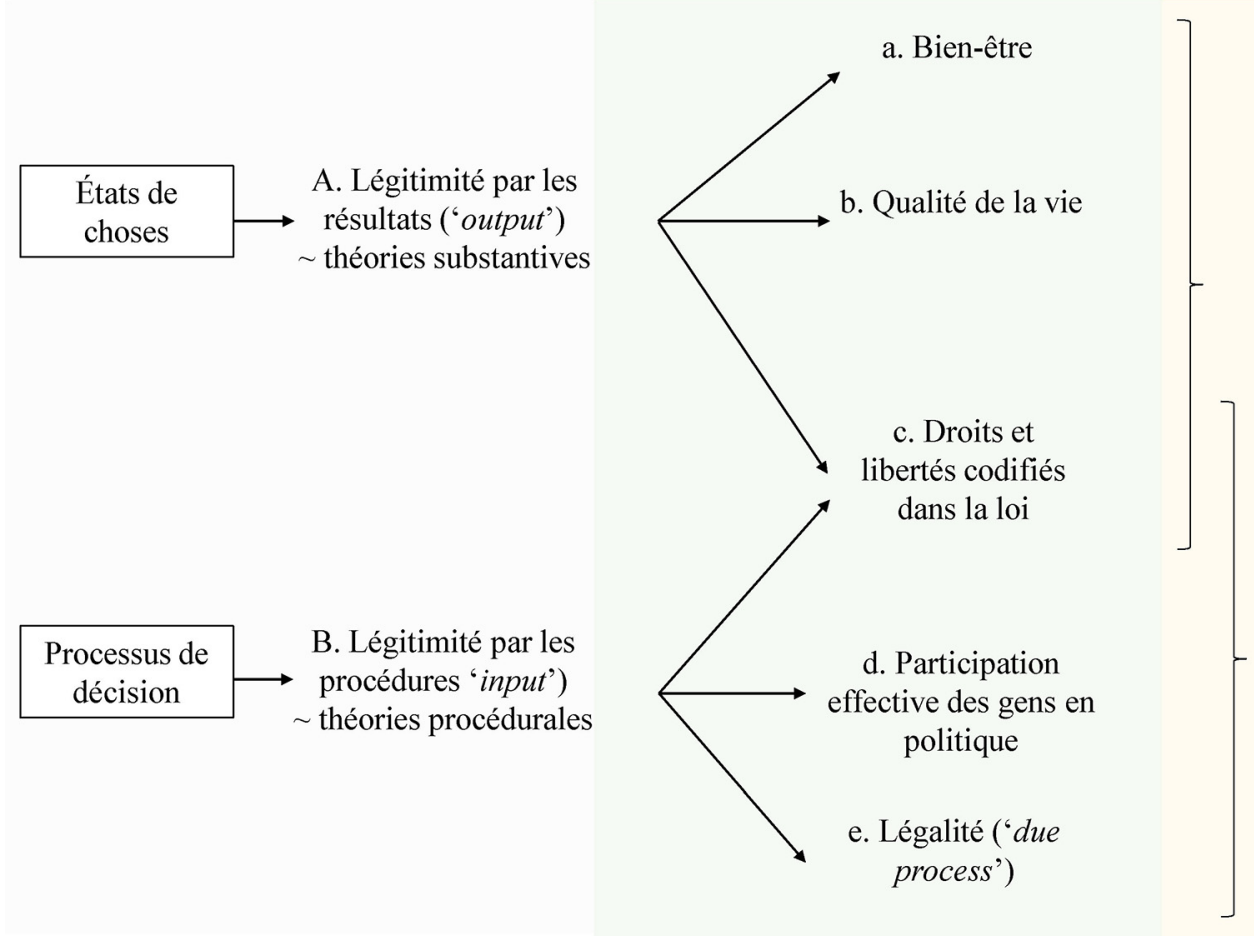

Quels sont les critères permettant d'attester de la légitimité ?
(1) Égalité de distribution
(2) Effectivité
(3) Efficacité
(4) Reconnaissance
Etc.

Fig. 1. Principales théories de la légitimité (d'après Meinard, 2017).

choisies (A. Légitimité par les résultats). Au sein de ces deux grandes approches, de nombreuses variantes existent. Ainsi, les approches de la légitimité par les résultats peuvent se focaliser sur les résultats en termes de bien-être (a.), en un sens héritier de l'utilitarisme des préférences (approches relevant du «welfarisme réel», au sens de Fleurbaey, 2003), ou sur des visions plus ambitieuses de ce qui détermine la qualité de la vie (b.) (Nussbaum et Sen, 1993), comme les capabilités au sens que Nussbaum (2000) donne à cette notion. De même, les théories de la légitimité par les procédures peuvent se limiter à examiner la légalité des procédures (e.) mais, plus souvent, elles posent des exigences plus ambitieuses sur les possibilités effectives de participation politique. De manière intéressante, certaines approches par les résultats ou par les procédures se retrouvent autour d'une exigence de garantie de droits et de libertés vus comme des résultats par les défenseurs de la légitimité par les résultats, et comme des garanties de participation future par les défenseurs de la légitimité par les procédures (Roussin, 2016). Enfin, la littérature contient d'innombrables variantes de chacune des approches ci-dessus, selon les critères considérés comme pertinents pour capturer les déterminants de la légitimité. Pour ne prendre que l'exemple des théories welfaristes réelles, on peut ainsi distinguer celles qui mesurent la légitimité à l'aune de la somme ou de la moyenne des utilités des individus (utilitarisme des préférences), celles qui la mesurent sur la base de la seule utilité atteinte par les individus les moins bien lotis («maximin» inspiré par Rawls, 1971), celles qui la mesurent en prenant pour critère l'absence d'envie entre individus (Arnsperger, 1994), etc.

Insistons sur un point capital de la lecture de cette représentation : elle ne spécifie pas si la notion de légitimité est comprise comme une notion positive ou une notion normative. En effet, à l'étape de choisir entre A. et B. (fig. 1), par exemple, on peut se poser deux questions bien différentes. La première, qui renvoie à la compréhension de la légitimité comme notion positive, est celle de savoir quels sont les éléments qu'il convient d'examiner pour savoir si la politique en question va être considérée comme légitime, dans les faits, par les personnes ou groupes concernés (en général, les membres de l'entité politique concernée et/ou les personnes ou groupes affectés par la politique examinée). La seconde question, qui renvoie à la compréhension de la légitimité comme notion normative, est celle de savoir quels sont les éléments qu'il convient d'examiner pour savoir si la politique en question doit être considérée comme légitime. De la même façon, à toutes les étapes du cheminement que l'on peut dessiner dans la figure 1, la question peut se comprendre en un sens positif ou en un sens normatif. En insistant comme nous le faisons ici sur le contraste entre une lecture positive et une lecture 
normative, nous ne voulons aucunement minorer l'importance des arguments, devenus classiques, sur la remise en cause de la distinction faits/valeurs (Putnam, 2002). La mise en évidence des apories dans lesquelles enferme la croyance en la possibilité de toujours établir une distinction nette entre le positif et le normatif est un acquis de la littérature qui ne nous semble pas discutable. Cet acquis ne contredit cependant aucunement l'idée selon laquelle un effort de clarification du statut, positif ou normatif, d'une question ou d'une théorie, peut apporter des éclairages conceptuels importants dès lors qu'il est exercé avec précaution.

Cette opposition entre une compréhension normative et une compréhension positive de la notion de légitimité nous semble importante dans la mesure où elle permet de distinguer les deux grands types d'approche qui, dans la littérature économique et philosophique récente, ont tenté d'englober la diversité des théories représentées dans la figure 1 en une théorie générale de la légitimité.

Le premier de ces deux types d'approche est issu de la théorie de la justification de Boltanski et Thévenot (1991). Cette théorie et ses prolongements cherchent à rendre compte de la diversité des visions de la légitimité dont sont porteurs les individus et les groupes, en identifiant les cadres de référence à partir desquels différentes justifications peuvent être élaborées. Ce type d'approche relève de ce que nous appelons une compréhension positive, car son objectif est de comprendre ce qui détermine qu'une politique est considérée comme légitime, dans les faits ${ }^{2}$.

Le second type d'approche, dont les expositions paradigmatiques sont dues à Rawls (1993), Habermas (1994) et Estlund (2008), relève de la littérature en philosophie politique analytique. Il consiste à ancrer la légitimité d'une politique dans l'acceptabilité des justifications dont elle fait l'objet et relève d'une compréhension normative: il s'agit d'identifier les justifications qui doivent être acceptées.

Ces deux grandes approches englobent toutes les variantes dont la figure 1 ne donne qu'une liste réduite. Dans les deux types d'approche, ces variantes apparaissent comme autant de ressources qui peuvent alimenter des justifications. La différence est que, dans l'approche issue de Boltanski et Thévenot (1991), les justifications possibles sont les justifications qui, de fait, sont portées par des individus ou des groupes. À l'inverse, dans l'approche de philosophie politique analytique, une question supplémentaire est posée: celle de savoir si les

\footnotetext{
${ }^{2}$ Dans le prolongement de la remise en cause de la distinction fait/valeurs, il est possible d'interpréter cette littérature en posant qu'elle assume un dépassement de l'opposition normatif/positif. Discuter de l'intérêt et des limites de cette interprétation dépasse les ambitions du présent article.
}

justifications qui, de fait, sont portées, sont ou non des justifications acceptables. Comme le montre Estlund (2008), dans cette expression, la notion d'acceptabilité doit être prise dans un sens contre-factuel et normatif. Il ne s'agit pas de savoir si la justification est acceptée dans les faits, ni si elle sera, dans les faits, acceptée. Ce dont il est question, c'est de savoir si elle doit, en un sens résolument normatif du terme, être acceptée.

En cohérence avec le positionnement normatif, et même prescriptif, que nous revendiquons dans ce travail, c'est en premier lieu à l'approche de philosophie politique normative que nous souhaitons nous rapporter. Cela étant, l'approche que nous avons appelée «positive» n'est pas exclue par cette revendication de normativité. En effet, le cadre posé par Boltanski et Thévenot (1991) évoque des «régimes» auxquels les acteurs font référence et des «tests» lors desquels les justifications qui peuvent être articulées en arguments relatifs à des éléments constitutifs de ces régimes sont mises à l'épreuve. L'examen des arguments utilisés dans les faits par les différents protagonistes de la mise en œuvre d'une politique donnée et leurs réactions vis-à-vis des arguments dont les autres protagonistes sont porteurs forment ainsi la matière d'une analyse positive de la légitimité. Parce que cette compréhension de la centralité des justifications et de leur articulation argumentative dans la légitimité des politiques nous paraît être un socle incontournable, partagé par les approches normatives et positives de la notion de légitimité évoquées plus haut, nous nous focaliserons, dans la suite de ce texte, sur les arguments utilisés par les acteurs (dans le prolongement des approches positives de la légitimité), tout en spécifiant des exigences normatives associées à l'idée d'argument (dans le prolongement des approches normatives qui structurent notre raisonnement).

\section{Acceptabilité et argumentation : exigences normatives associées à l'idée d'argument}

On voit, par le biais de cette vision de la légitimité que nous adoptons ici dans le sillage de la philosophie politique analytique, pourquoi le travail sur l' " argument » est avant tout une affaire de légitimation: s'il permet de structurer une justification acceptable des politiques environnementales, alors cet «argument» est légitimant. L'argument légitimant sera celui qui, selon la définition de Perelman (Perelman et Olbrechts-Tyteca, 1958), est un argument qui tend à convaincre plus qu'à persuader - c'est-à-dire à s'adresser à un auditoire constitué d'êtres de raison avec qui l'orateur mène une discussion plutôt qu'à des êtres passionnés avec lesquels il débat.

Afin de rendre transparent et cohérent ce cadre conceptuel, nous proposons d'utiliser une série d'exigences qu'il apparaît naturel d'associer avec l'idée 
d'argument ${ }^{3}$. Ce cadre est composé des trois exigences suivantes :

- E1. Un argument doit être transparent: un «argument» qui cache ses failles ou les ignore, ou qui se limite à donner une apparence argumentative à un rapport de force ou à la promotion d'un intérêt particulier, n'est pas un argument stricto sensu.

- E2. Un argument doit offrir un cadrage : un argument éclaire une situation, un fait ou un processus en mettant en évidence la logique principale à l'œuvre dans la problématique identifiée. Décider d'une grille de lecture fait apparaître le cas particulier comme l'exemplification d'une logique plus générale ou l'application d'une théorie.

- E3. Un argument doit être pertinent: il n'y a pas d'argument là où il n'y a pas de discussion, pas de position à prendre, pas d'action à entreprendre. Dans ces situations, un «argument» qui ne traite pas de la décision à prendre ou de l'action à réaliser, qui n'est donc pas pertinent, qui fuit la décision, n'est pas un argument stricto sensu.

Dans la partie suivante, nous allons confronter ce cadre normatif abstrait à la réalité concrète de notre cas d'étude.

\section{Les «boues rouges » : emploi vs environnement?}

\section{Une question de légitimité dans une crise politique}

Notre cas d'étude porte sur la décision d'accorder une dérogation sur le taux de produits toxiques rejetés en mer au large de Cassis, qui a occasionné le conflit politique et médiatique dit «des boues rouges». Ces produits sont issus de l'usine Alteo qui transforme la bauxite en alumine à Gardanne. Il y a cinquante ans, la solution adoptée pour gérer de trop grandes quantités de résidus, compte tenu du manque d'espace de stockage à terre, a été de construire une conduite débouchant sur la fosse de Cassidaigne. Au moment où cette décision a été prise, elle a engendré un important conflit local, finalement étouffé du fait du soutien de l'État au propriétaire d'alors, Pechiney (Loison et Pezet, 2006). Ces résidus formaient une boue rouge (couleur du fer oxydé) chargée en métaux

\footnotetext{
${ }^{3}$ De même que les notions de légitimité et d'aide à la décision, celle d'argument peut être comprise en un sens positif, plutôt que dans le sens normatif qui nous intéresse. Au sens positif, l'argument est un outil de rhétorique. Au sens normatif que nous adoptons ici, «bon argument» est un pléonasme. Ce qui permet de statuer sur le statut d'argument d'une portion de discours est le fait qu'il satisfait ou non aux exigences normatives (en l'espèce: E1-E2-E3).
}

lourds et métalloïdes (aluminium, arsenic, cadmium, titane). En 1996, Corinne Lepage avait réussi à imposer un décret ${ }^{4}$ demandant un plan d'arrêt de tout rejet à échéance de 20 ans. En 2012, lors des négociations précédant la création du parc national des Calanques (PNCal), dont le cœur accueille l'extrémité de la conduite, Nathalie Kosciusko-Morizet avait annoncé la fin des rejets. La dispersion des boues, qui devaient théoriquement rester au fond du canyon de Cassidaigne, s'étendait entre Port-de-Bouc et Hyères (Moreau et Dubuquoy, 2016).

À partir de cette période, la fin de la dérogation s'approchant, de nouvelles négociations ont débuté. Les retards dans la mise aux normes étant trop importants pour espérer la fin des rejets, la demande de l'État s'est transformée ${ }^{5}$ : non plus cesser tout rejet, mais cesser les rejets solides ${ }^{6}$. Cela était en cohérence avec les investissements de l'usine dans des filtres-presses, et était justifié par la préfecture en se référant aux listes de «meilleures techniques disponibles ${ }^{7} »$, prenant en compte en parallèle les contraintes financières de l'usine.

Cette orientation suivait la recommandation du conseil scientifique du PNCal qui, en 2014, avait proposé que la dérogation soit reportée, en imposant une surveillance très serrée à l'usine pour pousser à la réduction des pollutions résiduelles. Si l'on en croit certains membres du conseil, cet avis est issu d'une comparaison entre gravité des impacts écotoxicologiques et emplois préservés. Cette recommandation, confirmée par le conseil d'administration du PNCal, a enclenché une

\footnotetext{
${ }^{4}$ Décret 96-191/44-1994A du $1^{\mathrm{er}}$ juillet 1996, cité dans l'arrêté 166-2014A du 28 décembre 2015, www.actu-environnement. com/media/pdf/news-25957-arrete-alteo.pdf.

${ }^{5}$ Selon les administratifs et spécialistes scientifiques des Installations classées pour l'environnement, dans ces circonstances, la négociation est directe entre ministre de l'Industrie et industriels. Elle se fait en amont des prises de décision et cadre fortement les aspects pris en compte. Ainsi il nous a été impossible de comprendre comment la reformulation de la contrainte sur l'usine est apparue: aucun acteur rencontré n'en a été témoin.

${ }^{6}$ Décret n ${ }^{\circ} 2012-507$ du 18 avril 2012 créant le Parc national des Calanques, Journal Officiel, 0093, 19 avril 2012, 7048, article 22.

${ }^{7} \mathrm{La}$ meilleure technique disponible représente «le stade de développement le plus efficace et avancé des activités et de leurs modes d'exploitation, démontrant l'aptitude pratique de techniques particulières à constituer la base des valeurs limites d'émission et d'autres conditions d'autorisation visant à éviter et, lorsque cela s'avère impossible, à réduire les émissions et l'impact sur l'environnement dans son ensemble » (Directive 2010/75/UE du Parlement européen et du Conseil relative aux émissions industrielles [prévention et réduction intégrées de la pollution], Journal officiel de l'Union européenne, L 334/17, 24 novembre 2010).
} 
polémique. Ségolène Royal, ministre chargée de l'Environnement, a vivement réagi à travers un tweet. Une enquête publique a eu lieu et a été très controversée, tant du fait de son organisation, accusée de réduire le débat ${ }^{8}$, que de la personnalité du commissaire enquêteur ${ }^{9}$ et de son traitement des conclusions de l'enquête, jugé expéditif par certains (Barraqué, 2017).

Finalement, en décembre 2015, une dérogation a été accordée par le préfet pour six années supplémentaires, autorisant l'usine à rejeter à la mer des polluants à des taux supérieurs à ceux admis par la convention de Barcelone $^{10}$. La ministre chargée de l'Environnement a alors de nouveau réagi via twitter en accusant le Premier ministre d'avoir sacrifié l'environnement pour des emplois ${ }^{11}$.

Nous avons enquêté en 2016 sur les perceptions du problème par certains acteurs. Une quinzaine d'entretiens formels a été réalisée. Ils ont été enrichis par des observations de réunions, des manifestations, des discussions informelles, ainsi que l'analyse de trois reportages et documentaires produits cette année-là. Les acteurs pertinents pour ce travail ont été choisis parmi des hauts fonctionnaires locaux, des opposants locaux et nationaux au rejet, des représentants de l'usine et des scientifiques impliqués dans les comités interdisciplinaires sur l'environnement au sein desquels des connaissances scientifiques relatives à la question ont été produites (en particulier le conseil scientifique du PNCal, les observatoires Hommes-Milieux «bassin minier de Provence » et «Littoral méditerranéen »). Lors des entretiens, ce qui était recherché était la définition du problème perçu par l'enquêté puis, au fur et à mesure, les arguments «pour» ou «contre» la dérogation étaient présentés pour que l'enquêté y réponde. Dans cinq cas, une carte mentale a été produite afin de désigner les acteurs, lieux et entités concernés par le problème, les

\footnotetext{
${ }^{8}$ Bien que concernant le rejet en mer, principalement contesté par les habitants de la côte, la réunion finale a eu lieu à Gardanne, dans une salle si petite qu'à peine la moitié des personnes qui souhaitaient participer ont pu y entrer et que la presse en a été exclue.

${ }^{9}$ C'est l'ancien directeur de la DREAL Paca lui-même qui avait été désigné, et donc la personne qui était à la tête de l'instance que beaucoup considèrent comme responsable de n'avoir pas respecté les exigences d'amélioration appelées par le décret de 1996 (Barraqué, 2017).

${ }^{10}$ Arrêté 166-2014A du 28 décembre 2015, www.actu-envir onnement.com/media/pdf/news-25957-arrete-alteo.pdf.

${ }^{11}$ Voir Le Monde avec AFP, 2016. Les rejets de «boues rouges » jugés «pas acceptables» pour Ségolène Royal, Le Monde, 5 septembre, www.lemonde.fr/planete/article/2016/ 09/05/les-rejets-des-boues-rouges-a-cassis-juges-pas-accepta ble-par-s-royal_4992409_3244.html.
}

flux entre ces éléments, les enjeux portés par chacun, les solutions possibles pour résoudre chaque enjeu et les personnes qui soutiennent ou rejettent ces solutions. Ce protocole permettait à la fois de recueillir les arguments des acteurs rencontrés (en entretiens ou en contexte d'interaction scientifique ou politique) et l'image qu'ils se faisaient des raisonnements des autres acteurs.

Il est ressorti des enquêtes que la justification centrale des décisions politiques était l'argument de l'emploi, vu comme «argument économique». Que l'enquêté soit d'accord ou non avec l'idée que la rigueur réglementaire pourrait forcer l'usine à fermer, c'est cet argument qui était vu comme le plus légitime pour ceux qui avaient à décider. Il apparaît implicitement dans la recommandation du PNCal (conseil scientifique et conseil d'administration), qui a intégré l'idée que l'usine ne devait pas être fermée et ne devrait donc pas subir une demande d'investissements pour dépollution trop forte. Selon Ségolène Royal, ce serait aussi pour conserver l'emploi que le Premier ministre a ordonné à son préfet d'autoriser la dérogation, refusant de prendre en compte une opposition assez virulente ainsi que les arguments de sa ministre ${ }^{12}$.

Il est important de noter qu'au cours de la controverse, et en amont de la prise de décision du préfet, un grand nombre d'expertises a été commandé à des bureaux d'études - soit de façon réglementaire, soit à la demande de Ségolène Royal, qui voulait préciser les connaissances du moment sur les risques sanitaires, soit à celle des opposants à l'usine qui ont fait intervenir la Commission de recherche et d'information indépendantes sur la radioactivité (CRIIRAD). De 2014 à fin 2016, onze expertises, dont six avec mesures sur site, ont été produites en mer et autour du dépôt de boues à Mange-Garri. Or, parmi ces expertises, aucune ne porte sur des aspects sociaux ou économiques locaux ${ }^{13}$. D'un point de vue réglementaire, les autorisations d'exploitation d'installations classées ne se fondent que sur les meilleures techniques disponibles et les déclarations de contraintes économiques de l'usine, et la seule information obligatoire concernant les points de vue de la population est issue de l'enquête publique. Mais on peut souligner ici qu'aucune analyse sérieuse ou mesure fine sur ce sujet n'a été perçue comme nécessaire durant le processus politique pourtant houleux.

\footnotetext{
$\overline{{ }^{12} \text { Cet article du Parisien }}$ du 3 septembre illustre la tension, présente durant l'année 2016, autour de cette question au sein $\mathrm{du}$ gouvernement, www.leparisien.fr/societe/boues-rougesroyal-tacle-valls-03-09-2016-6090355.php.

${ }^{13}$ Ce manque d'enquête sera corrigé par une étude de l'Agence régionale de santé à Gardanne, durant l'année 2018, pour connaître les inquiétudes des habitants sur les questions sanitaires liées à l'entrepôt des boues à terre.
} 


\section{Un argument économique valable?}

Face à un argument, nous pouvons utiliser notre cadre d'analyse pour juger de sa validité.

Malgré la proéminence de cet argument dans l'espace public, le cadrage du problème par les acteurs du terrain ne se cantonnait pas du tout à un discours simple (aucun, d'ailleurs, ne souhaitait, en 2016, la fermeture de l'usine). La réponse en termes d'emplois n'était alors pas très opérante :

- D'une part, l'évaluation du nombre d'emplois liés à l'usine n'est pas claire - l'usine elle-même parle de 450 emplois et 200 chez les sous-traitants (Barraqué, 2017 ; communication de l'usine); d'autres y ajoutent une partie des transporteurs et des salariés du port autonome de Marseille par lequel transitent les énormes quantités de bauxite importées, puis d'alumine exportées; il n'est pour autant pas possible de connaître, sans mandat spécial pour récupérer les informations, la part exacte du temps de travail du port autonome concernée par ce transit. Il peut donc s'agir de 650 emplois comme de 1000. Le Parisien, quant à lui, l'estime à 650 dans l'article cité.

- La demande de mise aux normes, chez les opposants, n'impliquait en aucun cas le désir de fermer l'usine. Certains opposants à la dérogation proposaient même d'autres idées pour relancer l'usine de façon plus moderne (elle a une apparence très vétuste) et moins polluante pour la mer. L'un d'eux, par exemple, proposait la relocalisation de l'usine sur un autre site afin de résoudre les problèmes de contrainte foncière qui ont poussé aux rejets en mer par le passé - ce premier argument était défait par d'autres acteurs, notamment en raison d'un coût exorbitant. Ce coût estimé n'a bien sûr jamais été justifié ou documenté puisqu'aucune évaluation de faisabilité des autres solutions n'a été faite. Un autre opposant proposait de pousser plus encore la spécialisation de l'usine, en ne conservant que la zone «blanche» de l'usine (Mioche, 2011), celle où est traitée l'alumine pour devenir alumine de spécialité, et pas la «zone rouge » d'extraction de l'alumine à partir du minerai de bauxite. Cette usine est en effet une des très rares usines en Europe à produire de l'alumine de spécialité - or il n'est pas impossible d'appliquer le procédé de production à une alumine banale, ce qui apporte le plus de valeur et ne génère pas l'énorme quantité de résidus incriminés. On pourrait ainsi apporter de l'alumine et lui donner plus de valeur, plutôt que de partir du matériau brut, la bauxite. Ce dernier argument était en général adossé à des propositions de développement d'activités économiques " propres » et intégrées prenant en compte les synergies de production d'énergie entre différents équipements industriels du territoire.
Les acteurs portant ces deux derniers arguments tenaient à signaler en sus que le terme «emplois» est dans ce sens un abus car ce sont les «emplois visibles» ou «emplois médiatisés » qui intéressent les décideurs. Deux acteurs ont donné l'exemple d'emplois invisibles qui n'ont jamais donné lieu à des discussions publiques pour critiquer une loi. Selon eux, suite au décret de $2010^{14}$ concernant les tarifs d'achat aux particuliers et l'obligation de rachat par $\mathrm{EDF}^{15}$, la filière a perdu 35000 emplois en France. Pour autant, l'argument de l'emploi n'a jamais été opposé à la disparition de ce soutien au développement de l'énergie renouvelable. La différence entre emploi et emploi visible est en outre impossible à capter en termes économiques.

Une critique assez forte de l'argument de l'emploi est apparue plusieurs fois dans les entretiens. Le «reproche» central fait au traitement de la crise par l'État - du côté des militants comme des chercheurs (Barraqué, 2017) - est que les abus liés à des exceptions fiscales ne sont pas reconnus. Déjà, l'usine connaît un statut dérogatoire concernant ses taxes de pollution de l'eau - elle paie 3,5 millions d'euros au lieu de 12 millions du fait d'un amendement qu'a fait voter un député local (aujourd'hui, maire d'une commune voisine de Gardanne, qui se positionne très sévèrement contre l'usine).

Une autre analyse complète, très visible ${ }^{16}$ (mais qui n'a jamais été vérifiée par les chercheurs des comités d'expertise) est présentée par un militant, jeune retraité, ancien comptable chez Bolloré Europe. Il tend à affirmer de façon répétée que le découpage de l'activité de l'usine entre plusieurs entreprises permet de nombreuses occasions d'évasion fiscale, en particulier par la mise en déficit des branches dont le siège social est en France, tandis que celles qui sont à l'étranger et ne paient pas d'impôts en France s'affichent comme excédentaires. Si l'on devait faire un geste minimum dans le sens d'une réflexion scientifique économique sur la base de ces analyses, on pourrait évaluer le coût d'un emploi généré par l'activité de cette usine à plus de 10000 euros par an (de taxes non perçues), ce qui correspond à ce qu'a pu coûter une politique active de création d'emploi pour un

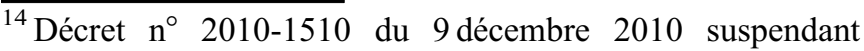
l'obligation d'achat de l'électricité produite par certaines installations utilisant l'énergie radiative du soleil, Journal officiel, 0286, 10 décembre 2010, 21598.

${ }^{15}$ Voir «Le moratoire sur les nouveaux projets photovoltaïques de décembre 2010», Photovoltaïque.info, www.photo voltaique.info/Decret-du-9-decembre-2010.html.

${ }^{16}$ Il s'agit de celle de Michel Mazzoleni, consultable sur son blog. Voir https://blogs.mediapart.fr/michel-mazzoleni/blog.
} 
secteur (le secteur de la restauration et l'impact de la loi de réduction de la TVA de 2009) ${ }^{17}$. Cela n'a jamais été présenté comme tel lors des justifications des choix par le Premier ministre ou par le préfet.

Globalement, cette analyse montre que l'argument de la sauvegarde de l'emploi, qui n'a été adossé à aucune démonstration en ce qui concerne la quantité réelle d'emplois et les coûts, se révèle particulièrement peu transparent et ne respecte pas du tout le critère E1 que nous avons posé.

À l'inverse, des arguments qui devraient être intégrés dans une analyse économique sont proposés par les acteurs locaux et peuvent à la fois soutenir l'usine ou critiquer la dérogation qui la protège encore jusqu'en 2021.

Contre l'usine, on trouve :

- la dangerosité des produits qui sont dorénavant stockés à terre au milieu de zones habitées et pourraient avoir un impact sur la santé, impliquant des coûts supplémentaires en termes de dépenses de santé (pris en charge par l'ensemble du corps social) et sur l'activité économique.

- le fait que l'usine a déjà plusieurs fois dépassé les taux de rejets admissibles dans le cadre de la dérogation dont elle a bénéficié (ce qui a été remarqué par des visites inopinées de la DREAL, mais n'apparaissait pas dans les autodéclarations de l'usine), ce qui signifie que la confiance n'est pas de mise et que la surveillance (coûteuse) des services de l'État doit être renforcée.

- les activités impactées en aval par la pollution de long terme sont de deux types : la petite pêcherie (elle n'est pas une activité économique très rentable, mais représente un patrimoine culturel important) et le tourisme qui repose, entre autres, sur ce patrimoine culturel et sur le patrimoine naturel du PNCal.

Pour soutenir le maintien de l'usine, deux arguments peuvent être raisonnablement pris en compte dans une analyse économique :

- l'usine a dû transformer ses savoir-faire dans les années 1990 , ce qui a occasionné une montée en compétences très forte des ouvriers ; les compétences de dépollution de l'usine sont en outre uniques au monde, la situation devient donc un laboratoire potentiel d'exemplarité pour le traitement des résidus

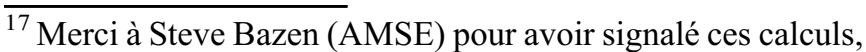
www.challenges.fr/challenges-soir/la-tva-restauration-ungachis-a-2-6-milliards-par-an_48103, avec une autre analyse sur la période 2004-2009 qui donne un coût par emploi d'environ 80000 euros de taxes non perçues (Bunel, 2013). Pour l'idée de la complexité méthodologique de ce type d'estimation, on peut se référer au numéro spécial de Travail et Emploi (Simonnet, 2014).
}

de la transformation de la bauxite. Encore faudrait-il que cet argument soit proposé à discussion;

- l'alumine technique de Gardanne est très utilisée dans des productions d'ordre stratégique (nucléaire, armement); d'autre part, l'usine se trouve sur le territoire, assurant ainsi une autonomie qui réduit des risques liés à des imports.

En ce sens, on voit que les dimensions économiques à prendre en compte sont bien plus nombreuses que les emplois visibles locaux, très difficiles à chiffrer; l'argument autour des emplois n'a donc pas la vertu de cadrage (E2) qu'auraient tous les enjeux économiques liés à la décision dans cet espace public.

Si l'argument économique était celui qui semblait le plus central dans la justification de la décision, constat que certains acteurs faisaient avec dépit, ce n'était pas pour autant le seul légitime, ni même celui qu'ils auraient eux-mêmes considéré comme pertinent. Si l'on reste dans le domaine purement politique, le déni de démocratie, les difficultés de certaines populations à faire entendre leurs droits, l'iniquité de traitement entre les différentes branches productives, le manque de politique industrielle cohérente étaient des préoccupations très présentes qu'il serait possible de mieux qualifier dans le contexte. Dans la perspective de pertinence (E3), on peut déplorer l'usage d'arguments qui, parce qu'ils se situent si loin des préoccupations exprimées, ont peu de chance d'établir la confiance du public et plus encore la légitimité du processus.

La hiérarchisation qui postule que des arguments économiques sont premiers dans la prise de décision, si elle est acceptée par le préfet, une partie des médias et des membres de comités locaux, n'est pas acceptée universellement, comme l'ont montré nos enquêtes. Mais, comme dit précédemment, jusqu'à très récemment, la mise à plat des inquiétudes, questions et arguments n'était pas de mise dans le conflit : l'enquête publique de 2015 a été très fortement critiquée dans son processus et sa synthèse; lors d'un comité de suivi de site de 2017, présidé par le sous-préfet (comité visant la circulation d'informations), les membres issus des associations ont quitté une réunion en bloc car ils considéraient que leur parole n'était pas écoutée (un médiateur a été nommé par la suite, ce qui a réduit les tensions). Le principal format de communication entre les opposants a été l'usage des médias (tweet, articles, films, blog) qui, systématiquement, polarisaient l'expression et ne laissaient pas la place au développement de contre-propositions ou d'analyses fines des enjeux (sauf le blog, malheureusement difficile à lire). Du fait du manque d'espaces pour organiser des discussions raisonnables et argumentées, le cadrage dénoncé par Ségolène Royal comme choix de sauver des emplois sans égard pour l'environnement s'est effectué bien en amont des décisions, au niveau 
national, et n'a plus évolué dans le débat local malgré des potentiels indéniables de discussion.

Lors de la prise de décision pour autoriser de façon dérogatoire le dépassement de seuils de pollution pour l'usine, en 2015, nous avons identifié que l'argument économique sous-jacent ou exprimé -l'emploi- ne répondait à aucun des trois critères que nous avons avancés :

- E1: Les seules études scientifiques effectuées pour l'aide à la décision sont des études techniques (comparaison de technologies, sanitaires et écotoxicologiques); l'argument essentiel de l'emploi n'a jamais été objectivé suivant les critères de scientificité minimum, à travers des comparaisons. Par exemple, si l'on choisit d'évaluer en emploi/coût l'impact de la fermeture du site (qui n'est qu'une des pistes proposées par les opposants), il s'agit de comparer deux situations : le maintien de l'activité (mesure rigoureuse des emplois directs et induits, coûts réels pour le contribuable du maintien de l'activité en fonction des dimensions déjà évoquées) et le contre-factuel choisi (coûts de cessation de l'activité, nombre d'emplois créés par le démantèlement et la dépollution du site) - cela implique que l'argument est grossier et ne présente aucune transparence.

- E2 : Le manque d'études ex ante par des économistes, tout autant que l'absence d'espace d'échange démocratique, n'a pas permis que se fasse une exploration des contre-propositions au statu quo ou à la fermeture de l'usine - l'argument de l'emploi, sans production de contre-factuels crédibles, ne suffit pas à cadrer le problème en définissant les bonnes limites à l'observation du système de production et son inscription dans le territoire.

- E3 : Jusqu'à aujourd'hui, la production experte autour de la controverse des boues rouges, qui a principalement suivi les obligations réglementaires (à l'exception d'une expertise de l'Agence nationale de sécurité sanitaire de l'alimentation, de l'environnement et du travail [ANSES] demandée par la ministre de l'Environnement), ne porte que sur des critères techniques et aucunement politiques ou sociaux, alors que des axes de réflexion autres que la classique dichotomie «emploi/environnement» étaient revendiqués par des opposants. L'administration s'est ainsi donné les moyens de ne pas répondre aux arguments des associatifs, ce qui rend peu pertinente l'aide à la décision mobilisée par ces expertises et l'intégration qui est faite dans les analyses par les commissions ou le conseil scientifique du PNCal.

\section{Conclusion}

Notre étude de cas nous a permis de mettre en évidence que l'argument principal qui a été fourni contre le respect de la réglementation, qui avait une apparence économique d'après les acteurs locaux, n'était pas un argument selon nos critères. En ce sens, la décision n'a pas été équipée par une aide à la décision légitimante, selon la policy analytics. Ce point théorique peut en outre être mis en regard de la grande agitation politique qui a duré pendant l'année 2016 dans la zone marseillaise sur ce thème. L'effet de ce manque de légitimité est, aujourd'hui encore, identifiable dans des réactions de militants face à l'administration : par exemple, lors d'une réunion de comité de suivi de site à la préfecture, en juin 2018, l'ensemble des associations a quitté la salle en se plaignant des démonstrations répétitives auxquelles elles étaient soumises, mais qui ne répondaient pas à leur inquiétude. En ne nous penchant que sur l'«argument économique», central et reconnu comme tel par les acteurs, afin de montrer son évanescence, nous constatons que la légitimité de la décision n'a en effet pas été assise sur cet argument.

Nous ne prétendons pas avoir apporté des preuves quantitatives du caractère généralisable de notre constat. Notre méthodologie relève de l'étude de cas et nous renvoyons à des contributions ultérieures l'examen de sa portée quantitative. Cela dit, si l'on accepte que les études de cas peuvent contribuer à l'avancée des connaissances, on peut voir que celle que nous avons menée dans ce travail suggère quelques idées qui méritent de plus amples explorations, dans la lignée de contributions émergentes dans la littérature académique récente.

Une première idée est que l'aide à la décision, en politique publique environnementale, apparaît très peu ancrée dans la science économique, sans attache ni avec le monde académique ni avec les connaissances de la science économique. Cette idée semble corroborée par certaines contributions à la littérature, au moins dans le cas des évaluations économiques environnementales. Ainsi, Laurans et al. (2013) montrent que peu d'études académiques d'évaluation économique environnementale présentent des utilisations effectives de telles évaluations dans la prise de décision. Marre et al. (2015) constatent de même que les prises de décisions concernant les écosystèmes marins et côtiers ne s'appuient guère sur les études académiques d'évaluation de ce type. Feuillette et al. (2015) s'inscrivent dans le même mouvement dans leur étude de l'utilisation des analyses coûts-bénéfices dans la mise en œuvre de la directive-cadre sur l'eau, en montrant qu'elles ne participent pas de la prise de décision. Enfin, Marcone (2017) souligne le faible rôle joué par les évaluations dans la mise en œuvre de la directive-cadre stratégie pour le milieu marin. De tels résultats vont dans le sens de l'hypothèse que nous formulons. Ils sont cependant restreints au cas des évaluations économiques, et Marcone (2017, p.22) souligne que, dans ce domaine, les publications qui s'intéressent à la question de la mise en œuvre effective sont rares. Il y a donc ici un champ 
d'investigation empirique important à explorer. Par ailleurs, au-delà de la question de la mise à contribution des productions académiques en science économique, notre analyse pointe des lacunes plus fondamentales, dont un manque d'objectivité dans la construction des éléments mis à contribution dans les débats. Cet aspect appelle de même des vérifications empiriques dans différents contextes de politiques environnementales.

La deuxième idée est que, malgré la faiblesse de son ancrage dans la science économique, le monde de la politique environnementale est submergé par le vocabulaire de la science économique, qui est largement réinvesti pour nourrir des discours qui apparaissent faire peu de cas des présupposés des connaissances économiques auxquels ils font référence. Ici encore, des travaux comme ceux de Marcone (2017), qui a analysé les arguments mis à contribution dans les débats à différentes étapes de la mise en œuvre de la directive européenne susmentionnée, apportent des éléments empiriques pertinents qui défrichent un champ d'investigation encore largement inexploré. En l'occurrence, les analyses de Marcone (2017, chapitre 3) pointent que, bien que nombre de notions économiques soient mises en exergue à plusieurs étapesclés de la structuration et de la justification des processus de décision, les arguments proprement économiques sont rares, peu précis et jouent rarement un rôle décisif dans les débats correspondants. De telles analyses demandent à être reproduites dans d'autres cas d'études et pour d'autres outils économiques afin de tester la portée de l'hypothèse que nous formulons.

Finalement, ces deux idées en suggèrent une troisième qui est que les politiques publiques environnementales voient leur légitimité mise à mal par l'appui qu'elles prennent sur des « aides » à la décision qui pèchent par leur manque d'ancrage scientifique.

Dans la perspective de déployer des tests quantitatifs de la pertinence de ces idées, rappelons que, comme indiqué en introduction, nous avons compris dans ce texte la notion d'aide à la décision en un sens très large, désignant l'appui qui est pris, à différentes étapes de l'élaboration et de la mise en œuvre des politiques concernées, par différents acteurs et sous différentes formes, sur des éléments de connaissance. Par opposition à cette notion générale d'aide à la décision, il serait important d'identifier plus précisément les processus d'aide à la décision, au sens de Tsoukiàs (2007), c'est-àdire les ensembles concrets d'interactions, circonscrits dans le temps et caractérisés par des ensembles définis d'acteurs et de contextes d'interaction, dans le cadre desquels les prétendus arguments économiques sont mis à contribution dans la genèse et la mise en œuvre de politiques environnementales bien précises. C'est à l'échelle de tels processus d'aide à la décision que les tests quantitatifs devraient être déployés. La nécessité de s'inscrire dans cette dynamique est d'ailleurs attestée par le fait que les différentes contributions évoquées cidessus s'appuient sur des typologies de modes d'utilisation des évaluations économiques (cas, en particulier, de Laurans et al., 2013, et de Marcone, 2017).

Par ailleurs, notons que les trois idées évoquées ont manifestement une portée bien plus large que les seules politiques publiques environnementales. Aussi faut-il souhaiter que la solidité des prétendus arguments économiques soit analysée, comme elle l'a été dans le présent texte, également dans d'autres domaines de la décision publique.

Au terme de notre raisonnement, le statut normatif, et même prescriptif, de notre discours rend nécessaire de clarifier le rôle que notre raisonnement prétend conférer à l'économiste. Deux clarifications, à cet égard, s'imposent afin de dissiper des craintes que notre raisonnement pourrait susciter chez le lecteur.

Premièrement, on pourrait craindre que notre raisonnement ne postule une autorité normative dont l'économiste serait dépositaire. Ce n'est pas le cas. Nous adoptons une posture normative car nous nous intéressons à la légitimité, prise en un sens normatif, mais aussi, plus fondamentalement encore, parce que notre objet est de penser l'aide à la décision, comprise en un sens normatif. L'économie entre en scène par la suite, car nous faisons le constat empirique que des discours qui se présentent comme des arguments économiques sont utilisés pour des tentatives de légitimation et d'aide à la décision.

Deuxièmement, et de manière liée, on pourrait être tenté d'inverser le raisonnement en se demandant si notre raisonnement n'en vient pas à affirmer que seul l'argument économique pourrait être à même de légitimer une politique. Ce n'est en aucun cas l'idée que nous portons. Notre argument ne s'ancre ainsi pas dans cette vision technocratique que la littérature (pensons, par exemple, à Callon et al., 2001) a désormais largement dénoncée, et qui fait de la connaissance scientifique, et plus précisément économique, le cœur de toute légitimité politique. Nous ne présupposons pas que la science économique est l'unique source, ni même qu'elle est une source, de légitimité; ce que nous défendons, c'est que l'apparence fallacieuse de science économique peut fragiliser la légitimité - tout comme, par exemple, des équations fallacieuses peuvent affecter la crédibilité d'un raisonnement scientifique, sans qu'il faille en conclure que les équations sont la seule source, ni même que ce soit une source tout court, de crédibilité scientifique. 


\section{Remerciements}

Une partie de ce travail a été financée par le Labex DRIIHM (dispositif de recherche interdisciplinaire sur les interactions hommes-milieux), programme «Investissements d'avenir » (ANR-11-LABX-0010) géré par l'Agence nationale de la recherche (ANR), l'observatoire HommesMilieux du bassin minier de Provence et le CNRS. Merci à Anne-Charlotte Vaissière pour ses commentaires et critiques sur une version antérieure du manuscrit.

\section{Références}

Arnsperger C., 1994. Envy-freeness and distributive justice, Journal of Economic Surveys, 8, 2, 155-186, doi: 10.1111/ j.1467-6419.1994.tb00098.x.

Barraqué B., 2017. Les boues rouges de l'usine PéchineyAlteo de Gardanne : de l'inertie à la toxicité, du rejet... et du dossier, Revue juridique de l'environnement, 42, 2, 273292, www.cairn.info/revue-revue-juridique-de-l-environne ment-2017-2-page-273.htm.

Boltanski L., Thévenot L., 1991. De la justification. Les économies de la grandeur, Paris, Gallimard.

Bunel M., 2013. Évaluer un dispositif sectoriel d'aide à l'emploi : l'exemple des hôtels-cafés-restaurants, Revue française d'économie, 28, 1, 73-121, doi:10.3917/rfe.131.0073.

Callon M., Lascoumes P., Barthe Y., 2001. Agir dans un monde incertain. Essai sur la démocratie technique, Paris, Éditions du Seuil.

Estlund D.M., 2008. Democratic authority. A philosophical framework, Princeton, Princeton University Press.

Feuillette S., Levrel H., Blanquart S., Gorin O., Monaco G., Penisson B., Robichon S., 2015. Évaluation monétaire des services écosystémiques. Un exemple d'usage dans la mise en place d'une politique de l'eau en France, Natures Sciences Sociétés, 23, 1, 14-26, doi: 10.1051/nss/2015004.

Fleurbaey M., 2003. On the informational basis of social choice, Social Choice and Welfare, 21, 2, 347-384, doi: 10.1007/s00355-003-0263-5.

Habermas J., 1994. Faktizität und geltung. beiträge zur diskurstheorie des rechts und des demokratischen rechtsstaats, Frankfurt am Main, Suhrkamp.

Laurans Y., Rankovic A., Billé R., Pirard R., Mermet L., 2013. Use of ecosystem services economic valuation for decisionmaking: questioning a literature blind spot, Journal of Environmental Management, 119, 208-219, doi: 10.1016/j. jenvman.2013.01.008.

Loison M.-C., Pezet A., 2006. L'entreprise verte et les boues rouges. Les pratiques controversées de la responsabilité sociétale à l'usine d'alumine de Gardanne (1960-1966), Entreprises et Histoire, 45, 4,97-115, doi: 10.3917/eh.045.0097.

Marcone O., 2017. Utilisation des évaluations économiques et émergence de conventions dans l'élaboration des politiques environnementales : le cas des Programmes de Mesures de la Directive-Cadre "stratégie pour le milieu marin " (DCSMM). Thèse de doctorat en sciences économiques, Brest, Université de Bretagne Occidentale.

Marre J.-B., Thebaud O., Pascoe S., Jennings S., Boncoeur J., Coglan I., 2015. The use of ecosystem services valuation in Australian coastal zone management, Marine Policy, 56, 117-124, doi: 10.1016/j.marpol.2015.02.011.

Meinard Y., 2017. What is a legitimate conservation policy?, Biological Conservation, 213, Part A, 115-123, doi: 10.1016/j.biocon.2017.06.042.

Mioche P., 2011. Alumine et risques industriels: le cas des boues rouges et des résidus, Travaux de l'obervatoire Hommes-Millieux du bassin minier de Provence, 1, www. ohm-provence.org/IMG/pdf/tohm-01_20115484.pdf.

Moreau L., Dubuquoy O., 2016. Zone Rouge, histoire d'une désinformation toxique, Paris/Ajaccio, Les Films d'ici/ France 3 Corse.

Nussbaum M.C., 2000. Women and human development. The capabilities approach, Cambridge /New York, Cambridge University Press.

Nussbaum M.C., Sen A. (Eds), 1993. The quality of life, Oxford/ New York, Clarendon Press/Oxford University Press.

Putnam H., 2002. The collapse of the fact/value dichotomy and other essays, Cambridge/London, Harvard University Press.

Perelman C., Olbrechts-Tyteca L., 1958. La nouvelle rhétorique. Traité de l'argumentation, Paris, Presses universitaires de France.

Rawls J., 1971. A theory of justice, Cambridge/London, Harvard University Press.

Rawls J., 1993. Political liberalism, New York, Columbia University Press.

Roussin J., 2016. Fonder la légitimité démocratique : conceptions majoritaires, constitutionnelles et épistémiques de la démocratie. Thèse de doctorat en philosophie, Paris, Université Paris 1.

Simonnet V., 2014. Évaluation des politiques actives du marché du travail, Travail et Emploi, 139, 3, 5-14, www. cairn.info/revue-travail-et-emploi-2014-3-page-5.htm.

Tsoukiàs A., 2007. On the concept of decision aiding process: an operational perspective, Annals of Operations Research, 154, 1, 3-27, doi: 10.1007/s10479-007-0187-z.

Tsoukiàs A., Montibeller G., Lucertini G., Belton V., 2013. Policy analytics, EURO Journal on Decision Processes, 1, 1-2, 115-134, doi: 10.1007/s40070-013-0008-3.

Citation de l'article : Meinard Y., Rouchier J. L'«argument économique» dans l'aide à la décision en politique environnementale et son évanescence : réflexions à partir du cas des «boues rouges» de Gardanne. Nat. Sci. Soc. 27, 4, 399-409. 